\title{
In memoriam Hendrik de Waard (1922-2008)
}

\section{Niesen}

Published online: 24 December 2010

(C) The Author(s) 2010. This article is published with open access at Springerlink.com

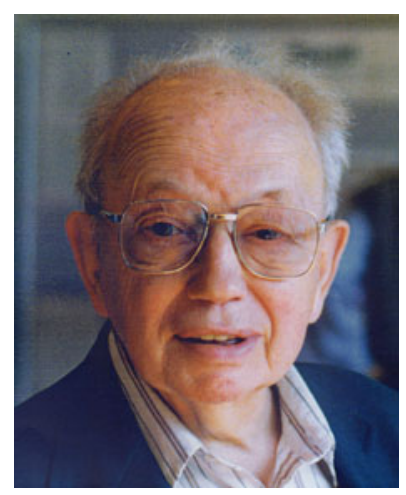

On august 11, 2008 Hendrik de Waard, one of the founding fathers of the hyperfine interactions community, passed away.

Born and raised in Groningen, he never left the place, except for short intermezzo's in Stockholm/Uppsala (1954-1955), Urbana (1962-1963), Murray Hill (1972-1973) and San Luis Obispo (several times). He studied at the University in his home town from 1940 till 1949, majoring in physics and mathematics. Needless to say there were other things to do during that period, and the Dutch resistance was one of them.

The end of World War II marked the start of tremendous activity in nuclear physics and quite naturally Hendrik became involved. His thesis work on nuclear spectroscopy on the isotopes ${ }^{181} \mathrm{Hf}$ and ${ }^{193} \mathrm{Os}$ lead to a doctorate in 1954 . His specialty was the measurement of short lifetimes, thanks to his deep knowledge of electronics and high-frequency phenomena. As an illustration: already before the war he attracted the interest of the local press for being able to receive, with homebuilt equipment, experimental TV emissions on the $80 \mathrm{MHz}$ band from the Philips engineers in Eindhoven.

L. Niesen $(\bowtie)$

University of Groningen, Groningen, The Netherlands

e-mail: 1.niesen@phys.rug.nl 
Back in Groningen after his stay in Sweden he became assistant professor in 1956 and full professor in 1958. He joined the excitement created when Lee and Yang suggested parity non-conservation in weak interactions, by building an instrument to measure the polarization of beta particles. Although he lost the race for the first experimental proof of parity non-conservation (the Nobel Prize winning experiment of $\mathrm{Wu}$ c.s. on oriented nuclei), the precise measurement of electron polarization remained an important activity in Groningen, mainly through the efforts of his student Johan van Klinken.

In 1962, as visiting professor in Urbana, Hendrik became in love with another phenomenon that led to a Nobel Prize: the Mössbauer Effect. Quite typically, he did not join the ${ }^{57} \mathrm{Fe}$ gang but started the first investigations with the $27.8 \mathrm{keV}$ level in ${ }^{129} \mathrm{I}$, which turned out to be very fruitful despite the fact that the ground state is radioactive. Although not trained as a chemist, he quickly became an expert on the Mössbauer spectroscopy of iodine compounds and wrote several review papers on the subject.

In 1965, Hendrik started a program that would become his opus magnum: the study of hyperfine interactions on radioactive nuclei that were introduced in solids by means of ion implantation. It had become clear that large magnetic fields existed at the nuclei of nonmagnetic atoms when they were incorporated in a ferromagnetic substance. The study of this phenomenon was severely hampered by the fact that the atoms of most elements of interest were insoluble in iron or nickel. Hendrik used the available isotope separator in the lab to accelerate radioactive ions, separate them from their stable counterparts and finally shoot them into a ferromagnetic foil. In the first experiments, ${ }^{129 \mathrm{~m}}$ Te ions were implanted to measure the magnetic field at ${ }^{129} \mathrm{I}$ nuclei in Fe. Contrary to the expectations of many sceptical solid state physicists in Groningen and elsewhere, the spectra looked neat and showed a large hyperfine field of roughly $100 \mathrm{~T}$. There were other spectral contributions however, demonstrating that not all ions (atoms) landed at regular lattice sites.

This result opened the possibility to explore the systematics of these hyperfine fields, utilizing as many experimental techniques as possible. In 1966 I became involved in the subject as a $\mathrm{PhD}$ student in Leiden, when Hendrik proposed to study radioactive ${ }^{133} \mathrm{Xe}$ nuclei implanted in $\mathrm{Fe}$ with the nuclear orientation technique. Shortly afterwards a package with radioactive foils was put on the train from Groningen to Leiden. On the outside it read: "fragile, do not drop" and the telephone number of the Kamerlingh Onnes Laboratory, to be dialled on arrival. I have to admit that the official in charge was quite suspicious when I came to collect this mystery package, but it worked! (Actually, it was not as bad as it may seem because the $81 \mathrm{keV}$ gamma radiation level was quite low at the outside).

Fortunately we did see a sizeable anisotropy in the emitted radiation when the foils were cooled to $15 \mathrm{mK}$, revealing an average hyperfine field of roughly $100 \mathrm{~T}$, just as in ${ }^{129} \mathrm{I} / \mathrm{Fe}$. When we communicated the preliminary results by phone, Hendrik reacted by sending a complete manuscript, in which we only had to fill in the final result!

The systematics and explanation of these hyperfine fields remained a large activity in the seventies. Apart from nonmagnetic elements, many of the rare earths were studied. Because several techniques yielded conflicting results, a lot of the Groningen effort was put in the development of other spectroscopic techniques like time-differential PAC (by Hohenemser and Pleiter) and NMR on oriented nuclei (by myself). 
As a second avenue, the investigation of various impurity-defect complexes produced by ion implantation became more and more important. These experiments disclosed a wealth of information on the behaviour of point defects, mostly vacancies and small vacancy clusters. Together with other spectroscopic techniques it revolutionized the knowledge of point defects in metals, which till then was largely based on the measurement of transport properties.

Ion implantation turned out to be extremely useful for doping semiconductors, and so the marriage with hyperfine studies on radioactive implanted ions was a logical one. Hendrik's work (with Dave Hafemeister) on ${ }^{129} \mathrm{Te} / \mathrm{I}$ implanted in Si was one of the first contributions in this field. Two components were found and associated with substitutional and interstitial sites, respectively. Later experiments using sophisticated annealing techniques showed this interpretation to be incorrect. While nearly all Te atoms could be brought to substitutional sites, the resulting spectra on the ${ }^{129}$ I daughter turned out to depend on the position of the Fermi level and showed the presence of at least three charge states for the I impurity. This system reveals how complicated the interpretation of source Mössbauer spectroscopy can be: the parent isotope governs the site in which the ions finally land, but the decay to the daughter creates a new situation in which trapping of charge carriers is of utmost importance.

Because of these complications Hendrik never really jumped into the semiconductor field. Instead he was fascinated by the possibility to make impurity clusters containing two foreign elements in a metallic host, something he coined "molecules in metals". Strong bonding was observed between several sp elements and halogens (especially F) when both elements were implanted into the same depth region. Although fascinating, this work never attracted a lot of attention, mainly because the studied molecules were the product of the nuclear decay, and therefore could not be produced easily in another way.

After his official retirement in 1986, Hendrik set up a collaboration with Richard Frankel (San Luis Obispo), an expert in magnetotactic bacteria. Apart from Mössbauer spectroscopy they studied the magnetic response of these small animals in time-dependent fields. It gave Hendrik the opportunity to build dedicated pieces of equipment, a hobby he enjoyed a lot.

I am very much aware of the fact that a description of his scientific career does not provide a complete picture of Hendrik de Waard. He was a multi-talented personality, who chose to be a University professor in physics, but could have chosen something completely different. When he was a student he seriously considered becoming an actor, not surprising for those of us who saw him acting on conferences on numerous occasions. But he was also the radio-amateur, getting in touch with his friends all around the planet. The science-politician, knowing exactly what he wanted and how to get there, social intelligence being the key factor.

Still that does not explain why the hyperfine interaction community was so fond of him. Maybe his secret was that whatever he did, a small glimpse of the young Groningen boy always radiated through. We will miss him a lot.

Open Access This article is distributed under the terms of the Creative Commons Attribution Noncommercial License which permits any noncommercial use, distribution, and reproduction in any medium, provided the original author(s) and source are credited. 